\title{
Estudio ecológico del swa (Carapa guianensis Aubl, Meliaceae) en tres comunidades del río Patuca, Honduras
}

\author{
Laura Díaz Arita ${ }^{1}$
}

Resumen. Carapa guianensis, swa (nombre en miskito) o cedro macho (nombre en español), es una especie nativa de los bosques húmedos tropicales de América. Su madera es muy útil y apreciada por los indígenas; de sus semillas se extrae un producto forestal no maderable llamado aceite de swa, que es medicinal y cosmético; es exportado por los indígenas a una empresa de productos para el cabello en Canadá. El estudio ecológico se llevó a cabo en las comunidades de Wampusirpi, Kurhpa y Krausirpi, en las vegas del río Patuca. Para ello, se midieron árboles de swa y árboles no swa que estaban dentro del transecto, para calcular variables ecológicas y estimar la capacidad de extracción de las semillas para la extracción del aceite y, con esta información, poder determinar las actividades de manejo para el uso sostenible, y evitar así, un futuro desequilibrio ecológico. Se entrevistaron a 30 productores de aceite para conocer la percepción ecológica que tienen acerca del recurso. En base a los altos resultados obtenidos en los transectos, sobre todo en la dominancia y el índice de valor de importancia de los árboles de swa medidos, se recomienda una extracción del $70 \%$ de las semillas que están en el suelo cálculo que se puede hacer al tanteo-, ya que el 30\% restante es necesario para la regeneración natural de los árboles de swa y la alimentación de animales silvestres.

Palabras clave: Aceite de swa, agroforestal, dominancia, extracción, índice de valor de importancia, producto forestal no maderable, regeneración natural.

\begin{abstract}
The Carapa guianensis, swa (name in miskito) or cedro macho (name in Spanish), is a native species of the humid tropical forests of America. Its wood is very useful and estimated by the natives, and from its seeds, a non forest wood product, called swa oil, is extracted, which is medicinal and cosmetic, and exported by the natives to a company of hair products in Canada. The ecological study was carried out in the communities of Wampusirpe, Kurhpa and Krausirpi, on the fertile plains of the Patuca river. Measurements of swa trees, as well as of other trees, were made, with the purpose of calculating ecological variables and estimating the capacity for extracting oil from the seeds, and with this information to be able to determine activities for managing their sustainability and avoiding any future ecological imbalance. Thirty oil producers were interviewed in order to find out their points of view on the resource. Based on the high results obtained in the transects, especially on the dominance and the importance value index of the swa trees measured, and extraction of $70 \%$ of the seeds on the ground is recommended, leaving the other $30 \%$ for the natural regeneration of the trees and for food for the wild animals.
\end{abstract}

Key words. Agroforestry, dominance, extraction, index of value of importance, non forest wood product, natural regeneration, swa oil.

\section{Introducción}

Los productos forestales no maderables son insumos utilizados ancestralmente por los pobladores de La Mosquitia hondureña, ya sea para extraer aceites, preparar ungüentos, pomadas medicinales $\mathrm{o}$ como alimento. Estos recursos son extraídos de la flora del bosque: de resinas, látex, hojas, semillas, frutos, cortezas, raíces, entre otros; y han sido y seguirán siendo de vital importancia para la sobrevivencia de las poblaciones indígenas y una parte fundamental de sus costumbres. Tienen un inigualable valor medicinal, alimenticio y mitológico. Los indígenas los han utilizado desde que se instalaron en esas tierras ricas y productivas que constituyen La Mosquitia. Lastimosamente, algunos usos se han ido perdiendo, como es el caso del swa - que fue rescatado por la organización Moskitia Pawisa (MOPAWI), una Organización no Gubernamental para el desarrollo de la Mosquitia -, para la venta del aceite.

La pérdida en el uso tradicional de productos forestales no maderables se genera por diferentes

${ }^{1}$ Licenciada en Biología, Apartado Postal 1843, Tegucigalpa, Honduras. Correo electrónico: biocamaron@yahoo.com.mx 
causas como son la extinción de la especie, debido al avance de la frontera agrícola, o al cambio climático global, igual que la poca transmisión generacional de la información, debido a la inserción de nuevos productos en el mercado.

Este estudio se centra en el árbol de swa o cedro macho (Carapa guianensis Aubl, Meliaceae), dándole énfasis a la producción del aceite que sale de sus semillas. Según los conocimientos de los indígenas de la zona, es utilizado para matar piojos y parásitos del cuero cabelludo y la piel, ya que es muy amargo. Cabe hacer notar que las técnicas para extraer el aceite son muy difíciles y requieren de mucho trabajo, por esa razón, los pobladores, especialmente las mujeres, decidieron hace algún tiempo dejar de producirlo.

En 2006, MOPAWI inicia la comercialización de este aceite, con la motivación de ayudar al crecimiento económico de los indígenas generando alternativas económicas y laborales. Los pobladores de Wampusirpi, en el río Patuca, no se imaginaban que hacer el aceite del swa podía llegar a ser una fuente de ingresos, pero ahora están muy entusiasmados con este nuevo proyecto, que incluso les ha servido para organizarse y trabajar en la venta del producto. Implementar y desarrollar un proyecto productivo de este tipo es difícil y requiere de diversos estudios del proceso de extracción del aceite, así como en el área social, cultural, económico y ambiental.

Este estudio representa los primeros pasos hacia la sostenibilidad ambiental, y con ello, la exitosa producción del aceite de swa. Encontrar el valor económico de una especie forestal no maderable sirve de motivación para la investigación y conservación de dicha especie y su ecosistema.

\section{Materiales y Métodos}

La investigación se realizó en tres comunidades del río Patuca en La Mosquitia hondureña. Se eligieron las comunidades de Wampusirpi, Kurhpa y Krausirpi, ya que son las que cuentan con el mayor número de productores de aceite de swa. En Wampusirpi se midió un transecto de $150 \mathrm{~m}$ de longitud por $8 \mathrm{~m}$ de ancho, ubicado $10 \mathrm{~km}$ río abajo de Wampusirpi en un sector denominado Kua. En la comunidad de Kurhpa se midió un transecto de $200 \mathrm{~m}$ de largo por $8 \mathrm{~m}$ de ancho. Por último, se midió otro transecto de $200 \mathrm{~m}$ de largo por $8 \mathrm{~m}$ de ancho en el cerro Wimpi ubicado frente la comunidad de Krausirpi.

En cada transecto se midió el diámetro a la altura de pecho (DAP) de los árboles de swa y los no swa, colectando los árboles no identificados por los guías locales y todas las especies del sotobosque que presentaran flor $\mathrm{o}$ fruto. Una vez terminada las mediciones de las tres comunidades, se realizaron los siguientes cálculos para determinar el valor de importancia de los árboles de swa en la zona y la estructura del ecosistema de árboles de swa y no swa. Todos los cálculos se realizaron únicamente tomando en cuenta al swa. El resto de especies pertenecen al grupo que no es swa:

a- Dominancia: Para la dominancia fue necesario calcular el área basal, con la información obtenida del DAP de las muestras de los transectos (Krebs 2001).

Área basal $=\pi \mathrm{r}^{2}$

donde;

$\mathrm{r}=$ radio

Dominancia= ---------------------

b- Abundancia: Su objetivo es definir qué especies son las que tienen mayor presencia en el bosque (Krebs 2001).

$\mathrm{Aa}=$ abundancia relativa de la especie

$\mathrm{Aa} \times 100 / \mathrm{A}$

Donde;

$\mathrm{Aa}=$ número de individuos de la especie "a"

$\mathrm{A}=$ número total de individuos

c- Frecuencia: Parámetro que mide la regularidad de la distribución horizontal de cada especie en la ocupación de un área. A la vez, es un indicador de diversidad o de la complejidad florística de la asociación dentro de la comunidad vegetal. Es el porcentaje de las parcelas en que ocurre una especie 
dada, en relación con el número total de parcelas (Krebs 2001).

$\mathrm{Fa}=$ frecuencia relativa de la especie.

$\mathrm{Fa} \times 100 / \mathrm{F}$

Donde;

$\mathrm{Fa}=$ número de parcelas donde ocurre la especie "a" entre el número total de parcelas levantadas

$\mathrm{F}=$ suma de las especies absolutas

d- Índice de Valor de Importancia (IVI): Es un parámetro que estima el aporte o significación ecológica de cada especie en la comunidad. El valor máximo es $300 \%$, y mientras más se acerque una especie a este valor, mayor será su importancia ecológica y dominio florístico sobre las demás especies y es igual a la suma de la dominancia, la abundancia y la frecuencia (Krebs 2001).

Para determinar el porcentaje de extracción de las semillas para la extracción del aceite, se contaron las semillas caídas al pie de los árboles de swa y el número de plántulas de swa regeneradas. Aproximadamente dos de cada diez semillas de swa que caen al suelo se regeneran, las demás se convierten en materia orgánica $\mathrm{y}$ alimento para pequeños mamíferos y son muy pocas las plántulas que llegan a convertirse en árboles adultos, debido a la competencia por la luz solar en estos densos bosques húmedos de La Mosquitia. Por último, se entrevistaron 30 hombres y mujeres de las comunidades para poder determinar la percepción que ellos tienen acerca del uso $\mathrm{y}$ conservación del árbol de swa.

\section{Resultados}

Estado de conservación y amenazas del swa. El árbol de swa es muy común en los bosques húmedos tropicales de La Mosquitia. Es un árbol con un alto valor ecológico (Guariguata y Kattan 2002), por su gran tamaño sirve de refugio para aves, serpientes y mamíferos; es fuente de substrato para herbáceas epifitas como bromelias y orquídeas, y sirve de alimento para pequeños mamíferos como guatusas (Dasyprocta ruatanica), jagüillas o chanchos de monte (Tayassu pecari) y quequeos (Tayassu tajacu), durante los meses de mayo, junio, julio y agosto, ya que son los únicos meses del año que produce fruto.

Los bosques de swa son húmedos, con un gran dosel superior, lo que genera en las semillas que logran germinar, gran competencia por la luz solar (CATIE 2000). El swa se encuentra en asociación con otros árboles de gran importancia (Zamora 2000), encontrados en los transectos, como, la masica (Brosimun alicastrum Sw.) árbol que sirve de alimento para los indígenas, medicinales como el bálsamo (Myroxylum balsamum (L.) Harms) y el pronto alivio (Guarea kunthiana A.Juss) o artesanales como el tuno (Castilla tuno Hemsl).

Los sitios donde se realizaron los transectos son bosques en donde los indígenas (misquitos o tawahkas) recolectan las semillas. Ellos, ancestralmente, han usado esos recursos naturales de una forma muy sostenible, sólo sacando lo que necesitan, ya sea flora o fauna, y respetando los árboles más viejos y reproductores, y cuidando a los más jóvenes.

El estado de conservación del swa, en el área donde se realizaron los transectos, es aceptable, ya que hay árboles adultos de hasta $3.0 \mathrm{~m}$ de circunferencia y numerosas plantas jóvenes para la regeneración. Además, con el nuevo valor económico que han adquirido las semillas del árbol, la percepción de los indígenas de la zona ha cambiado, motivándolos a no cortar los árboles grandes para extraer madera ni hacer pipantes.

Una de las amenazas que se identifican para el swa y todas las especies de la zona es el avance de la frontera agrícola, y la extracción ilegal de madera, sobretodo porque los responsables de este gran peligro no son indígenas, sino los ladinos del interior, que no respetan ni entienden el valor que representan estos recursos para la sobrevivencia de los pueblos y de todo el país en general.

Otra futura amenaza que se ha identificado hasta el momento, es el posible surgimiento de monocultivos de esta especie, ya que desequilibraría el ecosistema frágil $\mathrm{y}$ amenazado como es el bosque húmedo tropical. No significa que una finca bien manejada de swa no pueda ser sostenible, claro que sería una futura alternativa para la extracción de madera y de semillas para el aceite, pero tendría que realizarse siguiendo todas las recomendaciones y considerando experiencias de otros países como Brasil, ya que en 
Honduras no existen experiencias en el cultivo de esta especie y requeriría muchos estudios antes de hacerlo en la zona.

Transecto 1: Wuampusirpi, Kua. Kua se localiza río arriba de la comunidad de Wampusirpi, en las tierras bajas húmedas a la orilla del río Patuca. La zona está rodeada de un bosque muy transitado por los pobladores locales, ya que por el caminan hacia sus cultivos, recogen leña e incluso cazan animales. Además, es un punto cercano para colectar las semillas del swa para extraer aceite.

El transecto medía $150 \mathrm{~m}$ por $8 \mathrm{~m}$. Su área comprendía un bosque con un amplio y alto dosel superior, con árboles jóvenes y sotobosque no muy denso, esto debido a la intervención del machete cuando se cosechan las semillas de swa y cuando se abren paso los pobladores para dirigirse a otras actividades.

La cantidad de árboles adultos (swa o no) encontrados fue impresionante, acotando que la mayor circunferencia medida fue de $182 \mathrm{~cm}$ y la menor de 20 $\mathrm{cm}$. Se midieron 61 árboles, de los cuales 31 eran swa y 30 eran de otras especies (Cuadro 1).

El swa tiene una presencia relativamente mayor, por lo que su dominancia y el valor de importancia son mayores, en comparación con las demás especies juntas. Estos resultados se pueden ver influenciados por la ubicación del transecto, ya que se tomaron los bosques con mayor presencia del árbol de swa, recomendado por los lugareños. Además, es una especie que no se usa para leña y que los pobladores prefieren esperar que el árbol tenga mayor tamaño para utilizar su madera.
El cuadro 2 muestra los resultados de estos $150 \mathrm{~m}$. Refleja el éxito del swa en el área de Kua, por lo que la extracción de las semillas es mayor en esta zona, ya que además de estar cerca de la comunidad de Wampusirpi la cantidad de árboles es alta.

La dominancia, la frecuencia y el índice de valor de importancia (IVI) del swa son mayores que las especies no swa; esto se debe al gran tamaño de los árboles de swa medidos y encontrados (Cuadro 1).

Transecto 2: Kurhpa. Río abajo de la comunidad de Kurhpa, atravesando parcelas agroforestales, se encuentra un remanente de bosque húmedo, aquí se colocó el transecto $2(200 \mathrm{~m} \times 8 \mathrm{~m})$. Los pobladores locales acuden al lugar para cazar, colectar hierbas medicinales y recoger semillas de swa. El bosque tiene una alta diversidad de especies, con árboles grandes y leñosos, además de un denso sotobosque.

Este transecto presentó una alta densidad de individuos en comparación con otros bosques de otras partes de Honduras, ya que se midieron un total de 107, de los cuales casi una tercera parte son árboles de swa (Cuadro 3). A pesar de eso, el swa presenta una alta área basal y dominancia, debido, sobre todo, a la gran circunferencia medida de los árboles de swa, hasta de $580 \mathrm{~cm}$, mientras que en el resto de las especies la mayor circunferencia fue de $220 \mathrm{~cm}$ (Cuadro 4). Esta alta dominancia del swa, con respecto a otras especies, demuestra su alto valor de importancia para el equilibrio del ecosistema en que se encuentra, demostrando que se puede usar el recurso, siempre y cuando se respete un porcentaje mínimo para la regeneración, descomposición; y para la alimentación de pequeños mamíferos.

Cuadro 1. Resultados del transecto 1, Wuampusirpi, Kua, La Mosquitia, Honduras.

\begin{tabular}{|c|c|c|c|c|c|c|c|c|}
\hline \multirow[b]{2}{*}{ Árbol } & \multirow{2}{*}{$\begin{array}{c}\text { No. de } \\
\text { individuos }\end{array}$} & \multirow{2}{*}{$\begin{array}{l}\text { Área } \\
\text { basal } \\
\left(\mathrm{cm}^{2}\right)\end{array}$} & \multirow[b]{2}{*}{ Densidad } & \multirow[b]{2}{*}{ Frecuencia } & \multicolumn{3}{|c|}{ Porcentaje } & \multirow[b]{2}{*}{$\mathrm{IVI}^{\&}$} \\
\hline & & & & & Dominancia & Densidad & Abundancia & \\
\hline Swa & 31 & 1804 & 31 & 1 & 57.3 & 66.3 & 50 & 173.6 \\
\hline No swa & 30 & 1342 & 30 & 1 & 42.7 & 33.7 & 50 & 126.4 \\
\hline
\end{tabular}

${ }^{\&} \mathrm{IVI}=$ Índice de Valor de Importancia

Swa= Carapa guianensis 
Cuadro 2. Especies encontradas e identificadas en el transecto 1, Wuampusirpi, Kua, La Mosquitia, Honduras.

\begin{tabular}{lclll}
\hline & No. de & \multicolumn{2}{c}{ Nombre común en } & \\
\cline { 3 - 4 } Nombre científico & individuos & Español & Miskito & Familia \\
\hline Virola koschnyi Warb. & 1 & Sebo sangre & Bának & Myristicaceae \\
Trichospermun grewiifolium Kosterm. & 2 & Majao & Wahpi & Tiliaceae \\
Tetragastris panamensis Kuntze & 4 & Kerosene, chascal & Sahkal & Burseraceae \\
Pterocarpus officinalis Jacq & 8 & Sangre blanco & Kawi & Fabaceae \\
Luehea seemannii Triana \& Planch. & 1 & Guácimo colorado & Kira & Tiliaceae \\
Inga sp. & 4 & Guama & Bríbrit & Fabaceae \\
Cespedesia spathulata Seem. & 3 & Tamarindo & Bul & Ochnaceae \\
Carapa guianensis Aubl. & 31 & Cedro macho & Swa & Meliaceae \\
Desconocido 1 & 1 & & Sanitailo & \\
Spondias spp. & 1 & Pájara & Páhara & Anacardiaceae \\
Desconocido 3 & 1 & & Sanitailo & \\
Desconocido 4 & 1 & & Sisnika & \\
Desconocido 5 & 3 & Higuerillo macho & & \\
\hline
\end{tabular}

Cuadro 3. Resultados del transecto 2, Kurhpa, La Mosquitia, Honduras.

\begin{tabular}{|c|c|c|c|c|c|c|c|c|}
\hline \multirow[b]{2}{*}{ Árbol } & \multirow{2}{*}{$\begin{array}{c}\text { No. de } \\
\text { individuos }\end{array}$} & \multirow{2}{*}{$\begin{array}{l}\text { Área } \\
\text { basal } \\
\left(\mathrm{cm}^{2}\right)\end{array}$} & \multirow[b]{2}{*}{ Densidad } & \multirow[b]{2}{*}{ Frecuencia } & \multicolumn{3}{|c|}{ Porcentaje } & \multirow[b]{2}{*}{$\mathrm{IVI}^{\&}$} \\
\hline & & & & & Dominancia & Densidad & Abundancia & \\
\hline Swa & 29 & 2163 & 29 & 1 & 41.6 & 27.1 & 50 & 118.7 \\
\hline No swa & 78 & 3035 & 78 & 1 & 58.4 & 72.9 & 50 & 181.3 \\
\hline
\end{tabular}

${ }^{\&} \mathrm{IVI}=$ Índice de Valor de Importancia

Swa $=$ Carapa guianensis

Transecto 3: Krausirpi, Cerro Wimpi. El cerro Wimpi está frente a la comunidad de Krausirpi, río abajo de la comunidad de Wampusirpi, poblada en su mayoría por tawahkas. Para llegar a los árboles de swa hay que cruzar el río Patuca en pipante y luego caminar unos 45 minutos. Los árboles están unos 400 msnm, mayor altura sobre el nivel del mar que los transectos anteriores. El bosque es usado para extraer leña, plantas medicinales, semillas de swa y para la cacería. Los pobladores de Krausirpi han sobrevivido gracias a los recursos del cerro y sus alrededores.

En el transecto 3 se contaron 125 individuos, de los cuales 36 fueron swa (Cuadro 5). La mayor circunferencia en los árboles de swa fue de $250 \mathrm{~cm}$, mientras que la mayor de las otras especies fue de 339 $\mathrm{cm}$, medida en un bálsamo (Myroxylon balsamum (L.) Harms), un árbol medicinal. Este transecto, ubicado en un bosque húmedo tropical, con un denso sotobosque y bromelias terrestres, fue donde se encontró el mayor número de individuos (36). Además, la dominancia e índice de valor de importancia son bastante altos, debido al gran tamaño de los árboles de swa encontrados (Cuadro 6).

\section{Potencial de extracción de los bosques muestreados} de las semillas de swa para extraer aceite. En las zonas de extracción existe un gran número de árboles de swa y que tienen un alto nivel de importancia para el ecosistema, además de ser una especie de alto valor económico y de utilidad por su preciada madera.

Pese a que los resultados demostraron la gran dominancia de swa, la extracción de sus semillas debe ser controlada y manejada, ya que su regeneración natural y habitual se puede ver afectada por diferentes parámetros de humedad del suelo y la luz solar. Además, hay muchos animales que se alimentan de ellas, ya que por su estacionalidad, es probable que exista escasez de otros alimentos. 
Cuadro 4. Especies encontradas e identificadas en el transecto 2, Kurhpa, La Mosquitia, Honduras.

\begin{tabular}{|c|c|c|c|c|}
\hline \multirow[b]{2}{*}{ Nombre científico } & \multirow{2}{*}{$\begin{array}{l}\text { No. de } \\
\text { individuos }\end{array}$} & \multicolumn{2}{|c|}{ Nombre común en } & \multirow[b]{2}{*}{ Familia } \\
\hline & & Español & Miskito & \\
\hline Apeiba membranacea Spruce ex Benth. & 1 & Peine de mico & Kum & Tiliaceae \\
\hline Astronium graveolens Jacq. & 2 & Ciruelillo & Quitacalzón & Anacardiaceae \\
\hline Byrsonima crassifolia Kunth. & 2 & Nancite & Krabo & Malpighiaceae \\
\hline Carapa guianensis Aubl. & 29 & Cedro macho & Swa & Meliaceae \\
\hline Castilla tuno Hemsl. & 2 & Tuno & Tuno & Moraceae \\
\hline Cespedesia macrophylla Seem. & 3 & Tamarindo & Slim & Ochnaceae \\
\hline $\begin{array}{l}\text { Dalbergia glomerata Hemls. } \\
\text { (Dalbergia tucurensis Pittier.) }\end{array}$ & 1 & Granadillo & Rúsut & Fabaceae \\
\hline Grias cauliflora L. & 3 & Tabacón & Bul de swampo & Lecythidaceae \\
\hline Guarea kunthiana A. Juss. & 7 & Pronto alivio & Awanka & Meliaceae \\
\hline Hernandia stenura Standl. & 3 & Guaco & Panolan & Hernandiaceae \\
\hline Hirtella media Standl. & 9 & Guabiluno & Tururia & Chrysobalanaceae \\
\hline Lonchocarpus rugosus Benth. & 2 & Chaperon & Arbaika & Fabaceae \\
\hline Myrciaria floribunda O. Berg & 2 & Pimientilla & Brum & Myrtacae \\
\hline Pterocarpus officinalis Jacq. & 2 & Sangre blanco & Kawi & Fabaceae \\
\hline Simaruba glauca DC. & 1 & Negrito & Súkum & Simaroubaceae \\
\hline Spondias mombin L. & 1 & Jobo & Pahra & Anacardiaceae \\
\hline Symphonia globulifera L.F. & 3 & Varillo & Samo & Clusiaceae \\
\hline Terminalia amazonia Exell. & 1 & Cumbrillo & Ihingsa & Combretaceae \\
\hline Tetragastris panamensis Kuntze. & 5 & Kerosene, chascal & Sahkal & Burseraceae \\
\hline Virola koschnyi Warb. & 3 & Sebo sangre & Bának & Myristicaceae \\
\hline Vochysia ferruginea Mart. & 1 & San Juan rojo & Duhurang & Vochysiaceae \\
\hline Desconocido 1 & 1 & Barba de jolote & & \\
\hline Desconocido 2 & 1 & Laurel macho & & \\
\hline Desconocido 3 & 1 & Manteco & & \\
\hline Desconocido 4 & 1 & Niño & Krabu yane & \\
\hline Desconocido 5 & 1 & & Waibon & \\
\hline Desconocido 6 & 1 & & Bulwayan & \\
\hline Desconocido 7 & 1 & & Dilman suku & \\
\hline Desconocido 8 & 3 & Chilion & & \\
\hline Desconocido 9 & 6 & Ramón & & \\
\hline Desconocido 10 & 1 & & Cusua saba & \\
\hline
\end{tabular}

Cuadro 5. Resultados del transecto 3, Krausirpi, Cerro Wimpi, La Mosquitia, Honduras.

\begin{tabular}{|c|c|c|c|c|c|c|c|c|}
\hline \multirow[b]{2}{*}{ Árbol } & \multirow{2}{*}{$\begin{array}{c}\text { No. de } \\
\text { individuos }\end{array}$} & \multirow{2}{*}{$\begin{array}{l}\text { Área } \\
\text { basal } \\
\left(\mathrm{cm}^{2}\right)\end{array}$} & \multirow[b]{2}{*}{ Densidad } & \multirow[b]{2}{*}{ Frecuencia } & \multicolumn{3}{|c|}{ Porcentaje } & \multirow[b]{2}{*}{$\mathrm{IVI}^{\&}$} \\
\hline & & & & & Dominancia & Densidad & Abundancia & \\
\hline Swa & 36 & 2906 & 36 & 1 & 41.1 & 29.3 & 50 & 120.4 \\
\hline No swa & 89 & 4157 & 89 & 1 & 58.9 & 70.7 & 50 & 179.6 \\
\hline
\end{tabular}

${ }^{\&} \mathrm{IVI}=$ Índice de Valor de Importancia Swa $=$ Carapa guianensis 
Cuadro 6. Especies encontradas en el transecto 3, Krausirpi, Cerro Wimpi, La Mosquitia, Honduras.

\begin{tabular}{|c|c|c|c|c|}
\hline \multirow[b]{2}{*}{ Nombre científico } & \multirow{2}{*}{$\begin{array}{c}\text { No. de } \\
\text { individuos }\end{array}$} & \multicolumn{2}{|c|}{ Nombre común en } & \multirow[b]{2}{*}{ Familia } \\
\hline & & Español & Miskito & \\
\hline Apeiba membranacea Spruce ex Benth. & 1 & Peine de mico & Kum & Tiliaceae \\
\hline Brosimun alicastrum Sw. & 1 & Masica & Pisba mairin & Moraceae \\
\hline Bursera simaruba Sarg. & 1 & Indio desnudo & Limsi & Burseraceae \\
\hline Carapa guianensis Aubl. & 36 & Cedro macho & Swa & Meliaceae \\
\hline Castilla tuno Hemsl. & 9 & Tuno & Tuno & Moraceae \\
\hline Cecropia sp. & 1 & Guarumo & Plang & Cecropiaceae \\
\hline $\begin{array}{l}\text { Dalbergia glomerata Hemls. } \\
\text { (Dalbergia tucurensis Pittier.) }\end{array}$ & 1 & Granadillo & Rúsut & Fabaceae \\
\hline Dialium guianense (Aubl.) Sandwith & 2 & Tamarindo & Slim & Fabaceae \\
\hline Garcinia intermédia (Pittier) Hammel. & 1 & Jocomico & Sakipa & Clusiaceae \\
\hline Grias cauliflora L. & 1 & Tabacon & Bul de swampo & Lecythidaceae \\
\hline Guarea kunthiana A. Juss. & 2 & Pronto alivio & Awanka & Meliaceae \\
\hline Hernandia stenura Standl. & 1 & Guaco & Panolan & Hernandiaceae \\
\hline Hirtella media Standl. & 7 & Guabiluno & Tururia & Chrysobalanaceae \\
\hline Lonchocarpus rugosus Benth. & 5 & Chaperno & Arbaika & Fabaceae \\
\hline Luehea speciosa Willd. & 1 & Guácimo & Tui kwirka & Tiliaceae \\
\hline Myroxylon balsamum (L.) Harms. & 1 & Bálsamo & Maharasa & Fabaceae \\
\hline Nectandra hihua Rohwer. & 1 & Aguacatillo & Kuhlang & Lauraceae \\
\hline Pauteria izabalensis Baehni. & 2 & Selillon & Tasmuk & Sapotaceae \\
\hline Pouteria reticulata Eyma. & 7 & Zapotillo & Ibans wainka & Sapotaceae \\
\hline Pterocarpus officinalis Jacq. & 1 & Sangre blanco & Kawi & Fabaceae \\
\hline Symphonia globulifera L. F. & 2 & Varillo & Samo & Clusiaceae \\
\hline Terminalia amazonia Exell. & 1 & Cumbrillo & Ihingsa & Combretaceae \\
\hline Tetragastris panamensis Kuntze. & 1 & Kerosene, chascal & Sahkal & Burseraceae \\
\hline Trichilia pallida Sw. & 7 & Kerosén blanco & Pampi & Meliaceae \\
\hline Virola koschnyi Mart. & 7 & Sebo sangre & Bának & Vochysiaceae \\
\hline Desconocido 1 & 1 & Manga larga & Yuhura & \\
\hline Desconocido 2 & 1 & Tambor & Guapla & \\
\hline Desconocido 3 & 1 & & Yakuta & \\
\hline Desconocido 4 & 9 & & Yanan & \\
\hline Desconocido 5 & 1 & & Bulluam & \\
\hline Desconocido 6 & 1 & Cajulan & & \\
\hline Desconocido 7 & 3 & Chilion & Tasmok & \\
\hline Desconocido 8 & 2 & Palma & & Arecaceae \\
\hline Desconocido 9 & 1 & & Siaya pihini & \\
\hline Desconocido 10 & 2 & & Pukuro & \\
\hline
\end{tabular}

Los conteos demostraron que un árbol de swa puede producir más de 2000 semillas en junio, julio y agosto, de las cuales sólo $2 \%$ llegaráN a ser un árbol adulto, ya que la mayoría se convierten en materia orgánica, en alimento para pequeños mamíferos y otro porcentaje se ve atacado por la larva de Hypsipyla ferrealis Hampson (Lepidoptera: Pyralideae), lo que limita las plantaciones puras. Además, no se ha trabajado en el mejoramiento genético de $C$. guianensis (CATIE 2000). Por lo tanto, se recomienda, para la elaboración y venta del aceite, una extracción aproximada de $70 \%$ de las semillas totales; medida que se puede calcular al tanteo, estimando $30 \%$ para la regeneración y alimentación de animales. 
La cantidad de aceite pedida por la empresa canadiense debe de ser controlada y medida, evaluando con la experiencia que se tiene hasta ahora cuántas semillas y área colectada es necesaria para producir el pedido requerido. Si la producción del aceite es sostenible con el área y semillas utilizadas actualmente, se puede continuar vendiendo esta cantidad; sin embargo, si el área de extracción y las semillas no son suficientes, se debe considerar disminuir la cantidad pedida.

Esta proporción de extracción, es una medida de manejo recomendada hasta que los árboles que han sido cultivados en parcelas agroforestales alcancen la edad reproductiva y los dueños de las parcelas usen sus semillas, sin necesidad de ir al bosque para conseguirlas.

Es necesario saber que la extracción es una técnica muy primitiva de uso de los recursos naturales y que a medida que pasó el tiempo, el ser humano domesticó las especies vegetales que consideró necesarias para su diario vivir. La domesticación es un proceso largo, pero en el caso de los productos forestales no maderables y maderables, es la alternativa más sostenible.

Se recomiendan cultivos agroforestales, para evitar el brote de plagas que pueden causar daños a los ecosistemas. En el caso del swa hay que evitar los monocultivos, ya que pueden generar el brote de $H$. ferrealis, cuya larva ataca las semillas y únicamente se controla con pesticidas. En el caso de Honduras, no existen experiencias con monocultivos de swa, pero sí existen casos exitosos en sistemas agroforestales con cacao en La Mosquitia.

Percepción, uso y manejo de los productores respecto al swa, expresados en las entrevistas y reuniones comunitarias. Se entrevistaron a 30 productores, de los cuales 25 eran mujeres y 5 hombres, en una edad promedio de 39 años y un rango de edad de 15 a 81 años. Todos los entrevistados expresaron que antes de la compra del aceite de swa por Ojon Coorporation (empresa canadiense), ellos no conocían el proceso de extracción del aceite y que nunca lo habían utilizado. Esto refleja que si no es por el incentivo económico no existiría la extracción de aceite de las semillas de swa. Actualmente, cada uno de ellos, produce de 2 a 19 litros (Brown y Den ujil
2007), utilizando para ello entre 300 a 500 semillas por cada litro, dependiendo del estado de conservación y tamaño de las semillas.

Además, expresaron que hasta el momento no utilizan ninguna técnica de manejo, pero que su percepción para la conservación de esta especie ha cambiado. Ahora recapacitan y piensan mucho antes de cortar un árbol para usar su madera, ya que obtendrán mayor provecho económico de las semillas.

Un dato interesante es que 24 de las mujeres entrevistadas pertenecen a las organizaciones locales de mujeres productoras de aceite de swa, mientras que los hombres no están organizados; es decir, no pertenecen a las organizaciones.

De los 30 entrevistados, 11 habían cultivado swa en sus parcelas de cacao o en el patio de sus casas (899 árboles sembrados en total), 19 no lo habían hecho, pero piensan iniciar pronto su siembra de swa. Con respecto a la variación de la producción de las semillas de swa, los entrevistados afirman que hubo más producción de semillas en 2006, pero no saben exactamente por qué. Son muy pocas las observaciones ecológicas de los entrevistados. Expresan que "es importante la conservación y el manejo de los bosques de swa, ya que además de ellos existen muchos animalitos del bosque que lo necesitan para vivir, como la guatuza, el quequeo, ratones y el chancho de monte".

\section{Discusión}

Los resultados de los tres transectos demuestran el éxito de los árboles de swa, además de ser determinantes en la estructura y ecología de los bosques de bajura en las vegas del río Patuca. Mostrando como las especies vegetativas juegan un papel muy importante en la economía y cultura de nuestros pueblos indígenas. El swa y la comercialización del aceite demuestran que existen técnicas o medidas de conservación y de uso de los recursos naturales, encontrando un equilibrio entre ambas sin sacrificar las necesidades humanas ni destruir por completo los recursos naturales. Casos como estos comprueban que sí existe y es medible el valor económico del bosque, y que se debe de utilizar como herramienta para la conservación.

El trabajo de campo y los resultados, conllevan a 
una serie de recomendaciones de manejo para los bosques de swa, es necesario determinar las áreas de extracción y definir el número de hectáreas, para posibilitar en un futuro la certificación en un mercado voluntario para la no deforestación de los bosques de swa. La educación ambiental con los productores de aceite de la zona se hace necesaria para que ellos aprendan a determinar el porcentaje de extracción recomendado, igual que motivarlos a generar reforestación con el árbol de swa y cultivarlos de una manera agroforestal, y respetar las plántulas de regeneración natural. Además, el hecho de que los hombres no pertenezcan a ninguna organización, para efectos de manejo de los recursos naturales, puede ser una debilidad, ya que en la mayoría de los casos, son ellos los que van al campo y son los que conocen más la ecología del swa; por lo tanto, en ellos recae la mayor parte de las recomendaciones de manejo.

Es necesario incluir en los planes de manejo nacionales del Parque Nacional Patuca, la extracción y comercialización de los recursos forestales no maderables. Se hace visible la necesidad de documentar este tipo de experiencias a nivel nacional.
A raíz de los resultados, se observa la poca investigación existente en Honduras, para encontrar alternativas económicas que propicien la conservación de los recursos naturales.

\section{Literatura Citada}

Brown, E. y Den ujil, M. 2007. El procesamiento del aceite de swa. MOPAWI. Wampusirpi. $14 \mathrm{p}$.

CATIE. 2000. Manejo de semillas de 100 especies forestales de América Latina. Vol.1. CATIE, Serie Técnica, Manual Técnico No. 41, pp. 63-64.

Guariguata, M. y Kattan, G. 2002. Ecología y conservación de bosques neotropicales. Editorial Tecnológica de Costa Rica. 691 p.

Krebs, J. 2001. Ecología estudio de la distribución y la abundancia. México D.F. Reproflo, S. de C.V. 753 p.

Zamora, N. 2000. Árboles de la Mosquitia hondureña. Turrialba, Costa Rica. 335 p.

Recibido para publicación el 3 de julio de 2008 